\title{
Credit Assignment in a Motor Decision Making Task Is Influenced by Agency and Not Sensory Prediction Errors
}

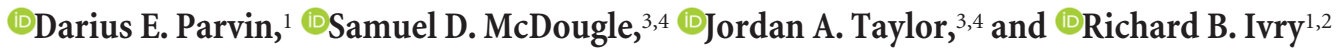 \\ ${ }^{1}$ Department of Psychology, ${ }^{2}$ Helen Wills Neuroscience Institute, University of California, Berkeley, California 94720, ${ }^{3}$ Department of Psychology \\ and ${ }^{4}$ Princeton Neuroscience Institute, Princeton University, Princeton, New Jersey 08544
}

Failures to obtain reward can occur from errors in action selection or action execution. Recently, we observed marked differences in choice behavior when the failure to obtain a reward was attributed to errors in action execution compared with errors in action selection (McDougle et al., 2016). Specifically, participants appeared to solve this credit assignment problem by discounting outcomes in which the absence of reward was attributed to errors in action execution. Building on recent evidence indicating relatively direct communication between the cerebellum and basal ganglia, we hypothesized that cerebellar-dependent sensory prediction errors (SPEs), a signal indicating execution failure, could attenuate value updating within a basal ganglia-dependent reinforcement learning system. Here we compared the SPE hypothesis to an alternative, "top-down" hypothesis in which changes in choice behavior reflect participants' sense of agency. In two experiments with male and female human participants, we manipulated the strength of SPEs, along with the participants' sense of agency in the second experiment. The results showed that, whereas the strength of SPE had no effect on choice behavior, participants were much more likely to discount the absence of rewards under conditions in which they believed the reward outcome depended on their ability to produce accurate movements. These results provide strong evidence that SPEs do not directly influence reinforcement learning. Instead, a participant's sense of agency appears to play a significant role in modulating choice behavior when unexpected outcomes can arise from errors in action execution.

Key words: adaptation; agency; credit assignment; decision making; reinforcement; reward

\section{Significance Statement}

When learning from the outcome of actions, the brain faces a credit assignment problem: Failures of reward can be attributed to poor choice selection or poor action execution. Here, we test a specific hypothesis that execution errors are implicitly signaled by cerebellar-based sensory prediction errors. We evaluate this hypothesis and compare it with a more "top-down" hypothesis in which the modulation of choice behavior from execution errors reflects participants' sense of agency. We find that sensory prediction errors have no significant effect on reinforcement learning. Instead, instructions influencing participants' belief of causal outcomes appear to be the main factor influencing their choice behavior.

\section{Introduction}

Consider the situation in which a tennis player attempts a passing shot, only to have her opponent easily return it with a winning volley. The player must decide whether the fault lies with her

\footnotetext{
Received Dec. 19, 2017; revised March 28, 2018; accepted April 7, 2018.

Author contributions: D.E.P. wrote the first draft of the paper; D.E.P., S.D.M., J.A.T., and R.B.I. edited the paper; D.E.P., S.D.M., J.A.T., and R.B.I. designed research; D.E.P. performed research; D.E.P. and S.D.M. analyzed data; D.E.P., S.D.M., J.A.T., and R.B.I. wrote the paper.

This work was supported by the National Institute of Neurological Disorders and Stroke-National Institute of Health, Grant NS092079 to R.B.I. and Grant NS084948 to J.A.T. We thank Matthew Boggess for help with data collection; and Faisal Mushtaq for helpful discussions.

The authors declare no competing financial interests.

Correspondence should be addressed to Dr. Darius E. Parvin, Department of Psychology, University of California, Berkeley, CA 94720. E-mail: darius@berkeley.edu.

DOI:10.1523/JNEUROSCI.3601-17.2018

Copyright $\odot 2018$ the authors $\quad 0270-6474 / 18 / 384521-10 \$ 15.00 / 0$
}

choice to hit a passing shot rather than a lob, or with her poor execution of the passing shot. How the brain solves this credit assignment problem, whether to attribute successes or failures to the selection or execution of actions, is poorly understood.

Reinforcement learning models that incorporate variables, such as reward magnitude and reward probability, have been quite successful in predicting choice behavior (Rescorla and Wagner, 1972) and associated neuronal activity (Schultz et al., 1997). Missing from this equation, however, is the role of action execution. These actions introduce a new set of variables to incorporate into the decision-making process, such as the effort required to make a particular choice (Walton et al., 2006; Hartmann et al., 2013) or the probability of successfully executing the required movement (Trommershäuser et al., 2008; Wu et al., 2009, 2011; Landy et al., 2012). However, current models typi- 
cally overlook the credit assignment problem, given the negligible role of motor errors in standard reinforcement learning tasks.

We recently considered how processes specific to action execution could provide information required to solve this problem (McDougle et al., 2016). We compared a traditional, buttonpressing "bandit task" with a modified version in which participants indicated their choices by reaching to one of two targets. In the former, the absence of reward provided information about the outcome probabilities associated with each stimulus (e.g., action selection error), whereas in the latter, the absence of reward provided information about reaching inaccuracy (e.g., action execution error), indicated by a visual cursor that landed outside the target. The results showed that participants' choice behavior was less sensitive to action execution errors compared with action selection errors. We proposed that this difference may have been due to the presence of a motor execution error signal in the reaching condition.

In the motor domain, sensory prediction errors (SPEs), the discrepancy between the predicted and actual sensory feedback, are used to correct the ongoing movements or to drive motor adaptation (Wolpert et al., 1995; Tseng et al., 2007). This signal could be directly exploited by the reinforcement learning system to solve the credit assignment problem. That is, the presence of an SPE could signal that the absence of the expected outcome (negative reward prediction error [RPE]) should be attributed to an error in movement execution rather than an erroneous choice. This "bottom-up" SPE hypothesis could provide a functional account of the relatively direct connections between the cerebellum, a critical component in the generation of SPEs, and the basal ganglia, parietal lobe, and orbital prefrontal cortex, core structures in reinforcement learning.

Alternatively, the credit assignment problem could be solved by a more "top-down" process related to a sense of agency, operationalized here as the belief that success or failure in obtaining a reward is determined by motor performance rather than the result of a property of the choices themselves. Green et al. (2010) proposed a model in which agency influences the rate of change in the values associated with response choices. In our reaching version of the bandit task, this would result in behavior consistent with discounting RPEs on trials with negative outcomes.

The current study further explores how action execution errors modulate reinforcement learning. The SPE hypothesis predicts that choice behavior should be sensitive to manipulations of the strength of the SPE, even if those manipulations are irrelevant to the reward outcomes. In contrast, the agency hypothesis predicts that manipulations of SPE strength should have a minimal effect on biases in choice behavior, and instead be influenced by the belief that the outcomes are dependent on their motor accuracy. Using a reaching variant of the two-armed-bandit task, we manipulated SPE by delaying reach feedback (Experiment 1), and by using "clamped" reaching feedback (Experiment 2). In Experiment 2, we also manipulated the task instructions to test whether biases in choice behavior were modulated by the participants' sense of agency.

\section{Materials and Methods}

Participants. All participants provided written consent, approved by the institutional review board at the University of California (Berkeley, CA). All participants were right handed, based on self-report and an assessment with the Edinburgh Handedness Inventory (Oldfield, 1971). Participants received either class credit or monetary compensation.

Experimental apparatus. Participants made reaching movements with their right arm on a graphics tablet $(49.3 \mathrm{~cm} \times 32.7 \mathrm{~cm}$, Intuos $4 \mathrm{XL}$;
Wacom, sampling rate $=200 \mathrm{~Hz}$ ) while holding a digitizing pen, embedded in a custom handle. The stimuli were presented on a monitor that was positioned above the tablet $(53.2 \mathrm{~cm} \times 30 \mathrm{~cm}$, ASUS). The monitor occluded the participant's view of their hand. The experimental software was custom written in MATLAB (The MathWorks) (RRID:SCR_001622) using the Psychophysics toolbox extensions (Pelli, 1997) (RRID:SCR_002881).

Reaching task. At the start of each trial, a white circle (diameter $1.2 \mathrm{~cm}$ ) was presented on the screen, indicating the start position (Fig. 1A). The participant was instructed to move their hand to the start location. Feedback of hand position was indicated by a solid white circle (diameter 0.5 $\mathrm{cm}$ ). This feedback was only visible when the hand was within $2 \mathrm{~cm}$ of the start position. After the cursor had been held in the start position for $1 \mathrm{~s}$, two red circles (diameter $1 \mathrm{~cm}$ ) were presented at a distance of $10 \mathrm{~cm}$, at $60^{\circ}$ and $120^{\circ}$ counter-clockwise relative to the right. The word "Go" appeared in the middle of the screen, instructing the participant to reach to one of the two circles. The participant was instructed to make a slicing movement, attempting to pass through the selected target. Cursor feedback was removed once the movement was initiated. If the reach amplitude did not reach $10 \mathrm{~cm}$ within $1.5 \mathrm{~s}$, the message "Please Reach Faster" was displayed and the trial was terminated. If the participant's reach deviated too far from either target (angular error $>20^{\circ}$ ), the message "Out of Bounds" was displayed. In both cases, the trial was immediately repeated.

If the hand passed within $20^{\circ}$ of the target, one of two trial outcomes occurred. On rewarded trials, the target color changed to green, a pleasant "ding" sound was played, and the number of points earned (1-100) was displayed above the chosen target. On unrewarded trials, the target remained red, an unpleasant "buzz" sound was played, and the number " 0 " was displayed above the chosen target. A box on the top of the screen showed the cumulative total of points earned.

Reward schedule. To assess target choice preference independent of reaching accuracy, the reward schedules were predetermined; as such, the outcomes were not contingent on whether or not the reaching movement actually intersected the selected target (with the exception of reaches judged to be out of bounds). Hit probability and reward functions were created using a bounded pseudo-sinusoidal function (Fig. $1 B$ ). These functions were mirrored for each target, such that the expected value for each target on a given trial was matched. For example, a "safe" target with a $90 \%$ hit probability and reward value of 10 points would be paired with a matching risky target that had a $10 \%$ hit probability and rewarded 90 points. We operationally define risk in terms of the probability of hitting the target. On hit trials, the participant received the associated reward value for that trial; on miss trials, no points were awarded. The probability and reward functions were designed so that at multiple points during the experiment, payoffs between the left and right targets gradually shifted, allowing us to track the participant's choice preferences. The same reward schedule was used for all participants, with the position of the targets counterbalanced.

Experiment 1. Experiment 1 was designed to compare conditions in which reach errors were signaled by a strong or weak SPE $(n=20$ per group; total $n=60,33$ female, age range $18-25$ years). At the location where the movement amplitude reached $10 \mathrm{~cm}$, the cursor reappeared, providing the participant with a feedback signal that indicated the accuracy of the reach (Fig. 1C). Presuming that the participant had intended to hit the target, the difference between the center of the target and the cursor position indicated the SPE for that trial.

Given that the hit/miss outcomes were predetermined, it was necessary to alter the feedback on some of the trials. On trials where the reach outcome matched the predetermined outcome, the reach feedback was veridical: The feedback cursor would fall on the target on hit trials $(22.5 \%$ of all trials) and off the target on miss trials (27.5\% of all trials). On trials where the reach outcome and predetermined outcome did not match, the reach feedback was manipulated. For "hits" that had to be converted to "misses" ( $25.5 \%$ of all trials), the cursor was displayed at a new location away from the target (in the same direction as the side of the target that was hit). To mask the fact that the feedback was sometimes altered, the distribution of the altered feedback signals was designed to closely match the distribution that results from variability in reaching, as determined in a pilot study (Fig. 2A). The new cursor location was randomly selected 
A

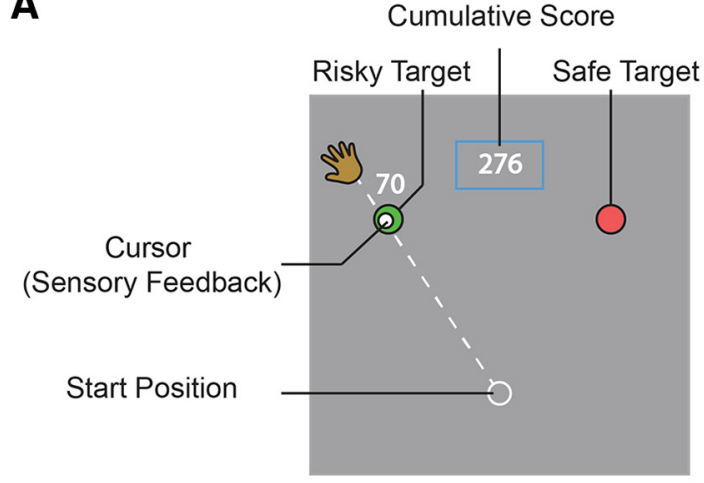

C

Experiment 1: Reach Feedback

Hit Trial

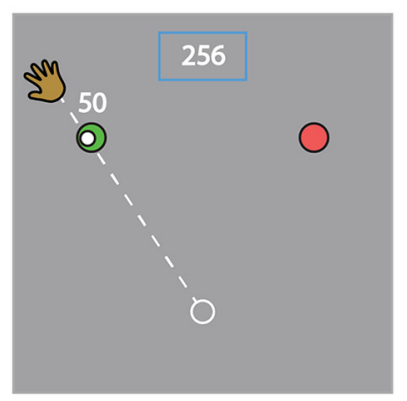

B

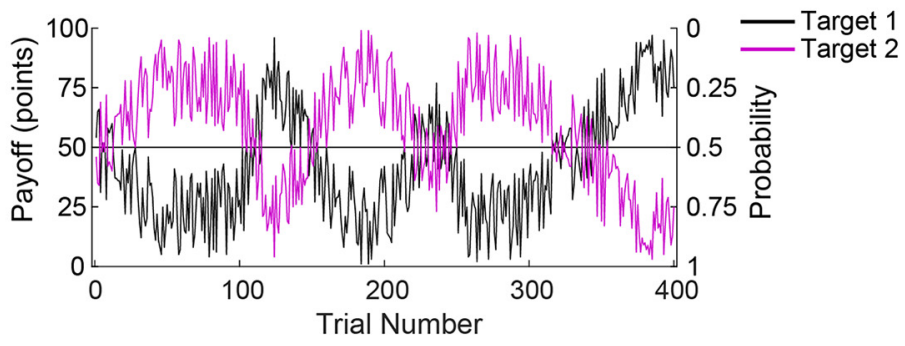

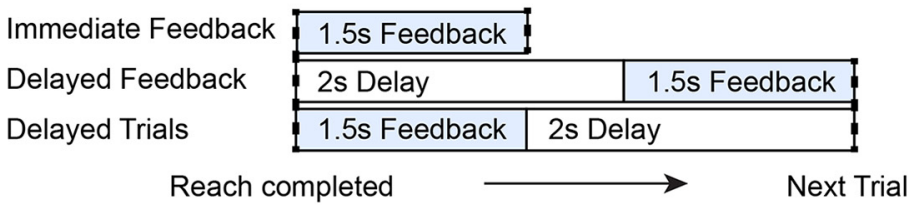

D
Experiment 2: Clamped Feedback (SPE+)

Hit Trial
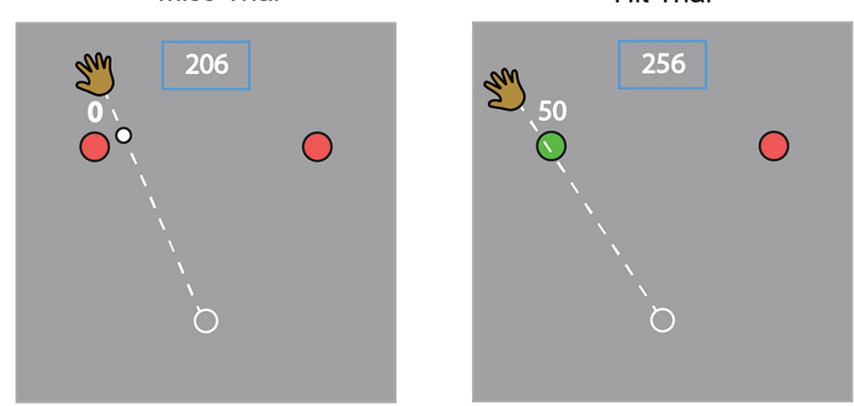

Miss Trial

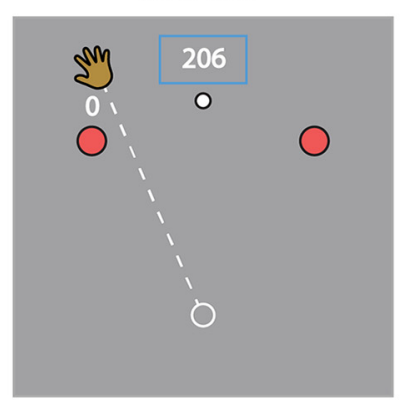

Figure 1. Experimental design. $\boldsymbol{A}$, Trials began with participants moving their hand to place the cursor at the start position. They indicated their choice preference by performing a shooting movement through the selected target. Visual feedback of the hand position was extinguished once the hand left the start position. In Experiment 1, visual feedback of the reach was provided on an imaginary circle with a radius equal to the target distance. On hit trials, the target would turn green and a pleasant "ding" sound was generated. On miss trials, the target would remain red and an unpleasant "buzz" sound was generated. The number of points earned was displayed above the chosen target (" 0 " in the case of a miss), along with a cumulative total of points earned displayed in a box. B, Top, Reward functions (left axis) and hit probabilities (right axis) for each target. Over trials, the targets vary in terms of their relative "risk" (e.g., high payoff but low hit probability) but are always matched in terms of the expected payoff. Bottom, Three groups were tested with different feedback delays and intertrial intervals. Immediate Feedback and Delayed Trials both received immediate reach feedback. Delayed Feedback received the same reach feedback but after a $2 \mathrm{~s}$ delay. C, Example feedback for hit and miss trials in Experiment 1 . Veridical feedback was provided when participants' actual accuracy (hit or miss) matched the predetermined outcome. For trials where they did not match, the cursor would be bumped in or out of the target on the same side, such that participants were not aware of the perturbation. $\boldsymbol{D}$, In Experiment 2, feedback of reaching accuracy was not provided. For SPE + groups, on miss trials, the feedback cursor was "clamped" and always presented at the same location between the two targets (regardless of which was chosen). On hit trials, no feedback cursor was presented. For SPE- groups, on all trials, no feedback cursor was presented.
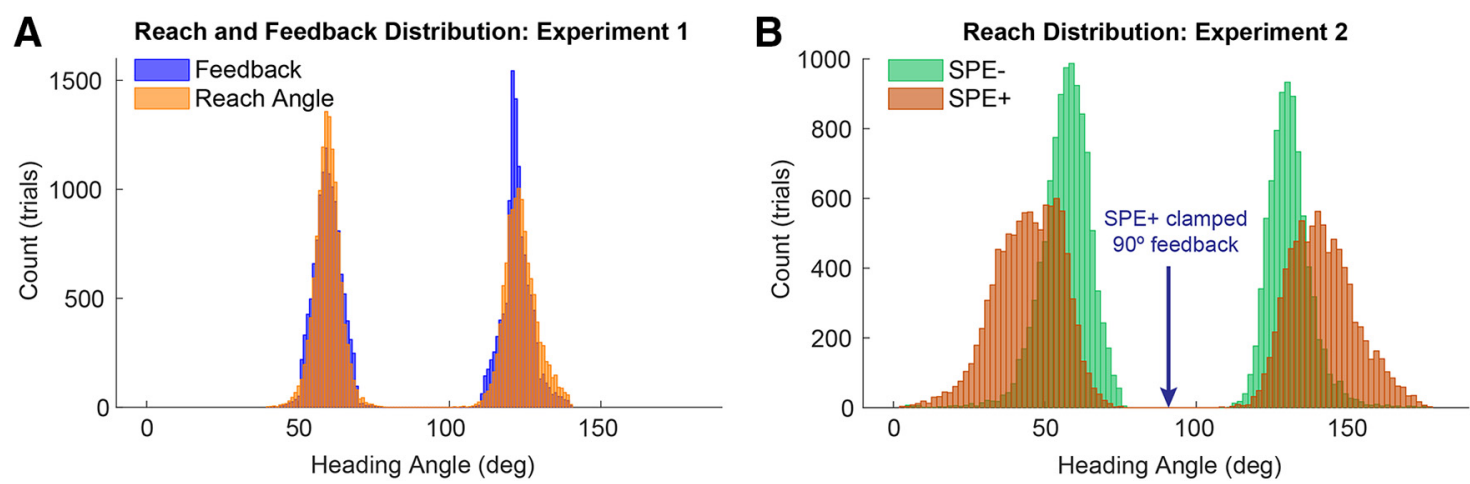

Figure 2. Distribution of reach endpoints and feedback location. $\boldsymbol{A}$, In Experiment 1, reach feedback was minimally altered to match the predetermined reward schedule. $\boldsymbol{B}$, In Experiment 2 , clamped feedback was provided at an invariant location $\left(90^{\circ}\right)$ on miss trials for the SPE ${ }^{+}$condition. As a result, the SPE ${ }^{+}$group heading angles are shifted away from the center relative to the SPE ${ }^{-}$ group, due to implicit adaptation.

from one side of a normal distribution with a SD of $4.65^{\circ}$, with the peak centered on the edge of the target. Locations deviating further than 2 times the distribution's SD $\left(9.3^{\circ}\right)$ were resampled. For "misses" converted to "hits" (24.6\% of all trials), the cursor was displayed within the target according to a uniform random distribution but restricted to the same side as the original miss. We included the "Out of Bounds" criteria to ensure that the feedback perturbations were relatively small, and thus prevent the participants from becoming aware of the feedback manipulation.

To manipulate the strength of the SPE signal, we varied the interval between the end of the reach and the time at which cursor feedback was 
provided. Previous studies have demonstrated that delaying sensory feedback by over $1 \mathrm{~s}$ can strongly attenuate the strength of an SPE (Held et al., 1966; Kitazawa et al., 1995; Honda et al., 2012; Brudner et al., 2016; Schween and Hegele, 2017). In the Immediate Feedback group, the cursor reappeared as soon as the reach amplitude exceeded $10 \mathrm{~cm}$ (Fig. 1B). In the Delayed Feedback group, the cursor feedback was presented after a $2 \mathrm{~s}$ delay. This manipulation confounds feedback delays and the time between successive trials. To unconfound these factors, we also tested a third group who received immediate cursor feedback but then had to wait an additional $2 \mathrm{~s}$ before the start of the next trial (Delayed Trials).

Experiment 2. In Experiment 2, we used a $2 \times 2$ factorial design $(n=20$ per group; total $n=80,51$ female, age range $18-25$ years). The first factor was to test whether an explicit sense of agency would alter participants' choice behavior. The second was to provide a second test of the SPE hypothesis.

In our previous study, we found no effect of agency (McDougle et al., 2016); however, our manipulation, which involved instructing participants that they were either in control or not in control of the hit/miss outcomes, may have been complicated by the inclusion of reach feedback in the vicinity of the target. The reach feedback may have unintentionally swayed participants to believe that they were still in control, regardless of the instructions. Here, we avoided this conflict by removing reach feedback completely. To manipulate a sense of agency, the participants were told that miss trials were either related or not related to their reaching accuracy. In the former case, the participants were told that the trial outcome reflected whether their reach accurately intersected the chosen target $\left(\right.$ Agency $\left.^{+}\right)$. In the latter case, the participants were told that the outcome reflected a probability that a target choice would result in a payoff, independent of their reaching accuracy $\left(\right.$ Agency $\left.^{-}\right)$. Beyond this instruction, the participants were not informed about the nature of the hit probabilities or reward schedule.

We also sought a second test of the SPE hypothesis, comparing conditions that did or did not include SPEs on miss trials. For participants in the $\mathrm{SPE}^{+}$conditions, we used a variant of task irrelevant clamped feedback (Morehead et al., 2017; Kim et al., 2018) to elicit SPEs without conveying reaching performance: On miss trials, the cursor feedback was always presented at a common location positioned between the two targets $\left(90^{\circ}\right)$ (Fig. $\left.1 D\right)$, appearing as soon as the reach amplitude exceeded $10 \mathrm{~cm}$. The participants were fully informed that, regardless of which target was selected, the feedback would always appear straight ahead on unrewarded trials. Given the instructions and lack of spatial correlation between the feedback and reaching movement (Fig. $2 B$ ), we assumed that these participants would not confuse the clamped feedback as indicative of their reach angle. Nonetheless, based on our previous work with clamped feedback of this sort, we assumed that these conditions would be sufficient to elicit SPE-dependent adaptation and, indeed, confirmed this in a separate "Clamp-only" control experiment (see "Clamp-only" experiment below).

Participants in the $\mathrm{SPE}^{+}$conditions received clamped feedback on all miss trials. This feedback signal was not presented to participants in the $\mathrm{SPE}^{-}$conditions. Neither group received cursor feedback on hit trials.

Experiment 2 block structure. The experiment consisted of 30 baseline trials, 400 decision making trials, and 30 aftereffect trials. The 400 decision making trials had the same reward schedule as Experiment 1. For the baseline and aftereffect trials, only one of the two targets were presented on each trial (location randomized), and the participant was instructed to reach to the target. A "ding" indicated that the movement amplitude had exceeded $10 \mathrm{~cm}$. No information was provided concerning reaching accuracy.

The baseline and aftereffect trials were included to assess whether the clamped feedback was treated by the motor system as an SPE. If so, the heading direction in the aftereffect block should be shifted in the lateral direction compared with the baseline block. Visuomotor adaptation was operationalized as a shift in heading angle in the aftereffect trials relative to baseline. The heading angle was defined as the angle between the hand position when it crossed the target radius, the start position, and the target. The heading angle values for the $60^{\circ}$ target (to the right) were flipped, such that for both targets, a positive heading angle represented the angle in the direction of expected adaptation (in the opposite direc- tion to the clamped feedback). All reported aftereffects were baseline subtracted, where the baseline was defined as the mean of all baseline trials.

Clamp-only experiment. The design and logic of Experiment 2 rests on the assumption that the clamped visual feedback is treated as an SPE (Morehead et al., 2017; Kim et al., 2018). Although the comparison of the baseline and aftereffect blocks in Experiment 2 provides a test of this assumption, we thought it prudent to conduct a clamp-only experiment that used a more traditional sensorimotor adaptation design, one in which the participants did not have to choose the reach target.

Reaches were made to a single target, displayed at either $60^{\circ}$ or $120^{\circ}$, the locations used in Experiments 1 and 2 (see Fig. 4A). The experiment consisted of 30 baseline trials (15/target) in which no visual feedback was provided, 120 "clamp" trials (60/target), and 10 aftereffect trials (5/target), again with no visual feedback. The trial structure was the same as in the baseline and aftereffects blocks of Experiment 2.

The clamp-only experiment also provided an opportunity to test the effect of delayed visual feedback on sensorimotor adaptation, relevant to our manipulation in Experiment 1. Two groups were tested (14/group, 14 female, age range 18-25 years), one in which the clamped feedback was provided coincidentally with the reach endpoint ("No Delay"), and a second in which the feedback was delayed by $2 \mathrm{~s}$ ("Delay"). If the clamp is treated as an SPE, adaptation should be evident in the "No Delay" group and abolished, or severely attenuated in the "Delay" group.

Statistical analysis. The chosen sample sizes were based on our previous studies using the reaching variant of the two-armed bandit (McDougle et al., 2016) and the clamp method (Morehead et al., 2017; Kim et al., 2018). All $t$ tests were two-tailed and used a threshold for significance $(\alpha)$ of 0.05 unless stated otherwise. We computed the inverse Bayesfactor $\left(B F_{01}\right)$ for our results from Experiment 1 to assess the likelihood of the null hypothesis $\left(H_{0}\right)$ relative to the SPE hypothesis $\left(H_{1}\right)$. We used a method proposed by Rouder et al. (2009), using a prior for effect size following a Cauchy distribution with a scale factor of 1 . Here, $B F_{01}<1 / 3$ can be considered as strong evidence in favor of the alternative hypothesis, $B F_{01}>3$ as strong evidence in favor of the null hypothesis, and anything between is only considered weak or anecdotal (Dienes, 2014).

\section{Results}

\section{Experiment 1}

In Experiment 1, we set out to test the SPE hypothesis, the idea that the operation of the reinforcement learning system is attenuated following trials in which the absence of a reward is attributed to an error in action execution rather than action selection. The core prediction of this bottom-up hypothesis is that the strength of the SPE signal should influence choice behavior. Participants were tested in a two-armed bandit task, indicating their choices on each trial by reaching to one of two targets. In addition to receiving reward feedback, cursor feedback indicated the accuracy of the reach. We compared two groups, an Immediate Feedback group who saw the feedback cursor immediately at the end of the reach; and a Delayed Feedback group, for whom the appearance of the feedback cursor was delayed by $2 \mathrm{~s}$. Based on previous studies, the strength, or salience of SPE should be considerably attenuated in the Delayed Feedback group (Held et al., 1966; Kitazawa et al., 1995; Honda et al., 2012; Brudner et al., 2016; Schween and Hegele, 2017). Given that the 2 s feedback delay also increases the time between successive trials, we also tested a Delayed Trials group in which the feedback cursor appeared immediately at the end of the reach, but with an extra $2 \mathrm{~s}$ pause between trials. In this manner, we matched the trial-to-trial interval of the Delayed Feedback and Delayed Trials groups.

In standard bandit tasks in which the outcome is not dependent on action execution, people typically show a preference for the "safe" target, consistent with a risk aversion bias (Kahneman and Tversky, 1979; Niv et al., 2012; McDougle et al., 2016). In a previous study (see also Wu et al., 2009; McDougle et al., 2016), we observed 
A

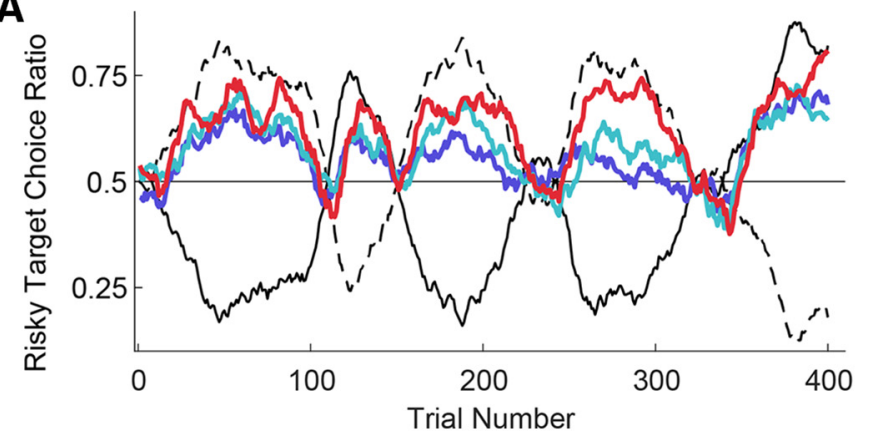

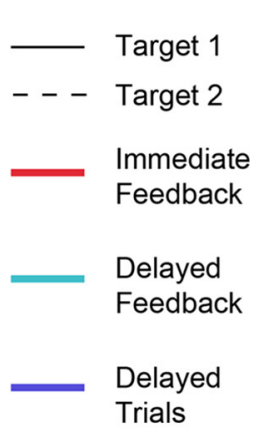

B

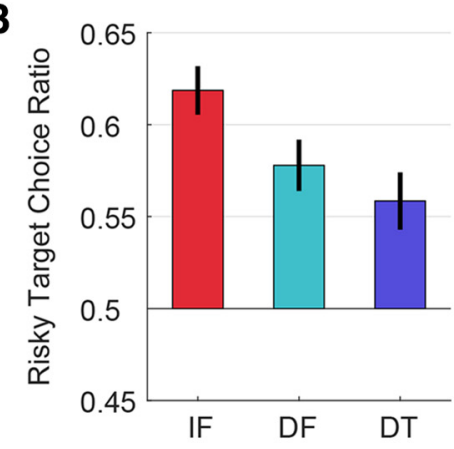

Figure 3. Increasing the trial-to-trial interval, either by delaying feedback or increasing the intertrial interval resulted in a weaker preference for the risky target. $\boldsymbol{A}$, Mean group choice behavior reveals overall preference for riskier target throughout the experiment. Colored lines indicate the proportion of choices made to the riskier target, averaged over participants in each condition (calculated over a 19-trial window moving average). The relative "riskiness" of target 1 and target 2 (determined by the predefined reward schedule) are shown for illustrative purposes (black solid and dashed lines). $\boldsymbol{B}$, Risk preference quantified as the ratio of trials where the riskier target was chosen over the total number of trials. All groups exhibited a preference for the riskier choice ( $>0.50$ ), with this effect significantly greater for the IF group compared with the other two. IF, Immediate Feedback; DF, Delayed Feedback; DT, Delayed Trials. Error bars indicate \pm 1 SEM over participants.

a striking reversal of this preference when the choices were indicated by reaches, so that the failure to obtain a reward was attributed to a failure of action execution. The SPE hypothesis predicts that this reversal is due to the presence of SPEs on miss trials. Consistent with those results, the Immediate Feedback group and Delayed Trials group showed a consistent preference for the riskier target over the course of the experiment (Fig. 3A). However, in contrast to the SPE hypothesis, the Delayed Feedback group also showed a reversal of the risk aversion bias, even though we assume the strength of the SPE is greatly attenuated by the delay (an assumption we confirm in Clamp-only Experiment).

For each trial, we defined the risky target as the one with the lower hit probability, but higher payoff and, as such, the option with a larger variance of potential outcomes (Kahneman and Tversky, 1979; Caraco et al., 1980; Dayan and Niv, 2008; Schultz, 2016). Using this definition, we quantified participants' choice biases by calculating the ratio of trials in which they picked the riskier target over the total number of trials (excluding the few out of bounds trials). A one-way ANOVA revealed a significant effect of group on risk bias $\left(F_{(2,57)}=4.65, p=0.01\right.$; Fig. $\left.3 B\right)$.

Post hoc $t$ tests using Bonferroni-adjusted $\alpha$ levels of 0.017 $(.05 / 3)$ were conducted. A numerical but nonsignificant difference (after correcting for multiple comparisons) existed between the Immediate Feedback and Delayed Feedback groups $\left(t_{(38)}=\right.$ 2.13, $p=0.04)$. This difference is in a direction consistent with the hypothesis that SPE influences choice behavior. However, we observed a significant difference between the Immediate Feedback and Delayed Trials groups $\left(t_{(38)}=2.95, p=0.005\right)$, indicating that an increase in intertrial interval alone (i.e., without manipulating the SPE) affected choice preference. The Delayed Feedback group had a numerically lower risk bias compared with the Delayed Trials group, opposite to what the SPE hypothesis predicts, although this difference was nonsignificant $\left(t_{(38)}=0.93, p=\right.$ 0.36). An inverse Bayes factor comparing the odds of the hypothesis that the Delayed Feedback and Delayed Trials risk biases were equal (null) versus the hypothesis that they were unequal provided only weak support in favor of the null $\left(B F_{01}=2.95\right)$.

Together, these results fail to support the hypothesis that choice biases are modulated by the strength of the SPE. The most parsimonious interpretation of the current results is that choice biases in the current task decay as a function of the time between successive trials, independent of the strength of the SPE. This could be the result of time-sensitive processes, such as a decay of the representations of the value of the target, or decay of a motor memory that could be used to adjust the next movement (see Discussion).

\section{Experiment 2}

The results of the first experiment indicate that SPE is not a critical signal that directly modulates choice biases. An alternative hypothesis is that, due to the sense of agency associated with reaching (Green et al., 2010), people may be slow to update their estimates of action execution errors based on recent outcomes. For example, the participants have a strong prior for their reaching competency and believe that their execution errors simply reflect motor noise, a variable that should operate randomly across trials. We set out to test this hypothesis in Experiment 2, comparing conditions in which participants were told that the absence of reward was attributed to a failure in motor execution (Agency ${ }^{+}$) to conditions in which the absence of reward was attributed to a property of the object $\left(\right.$ Agency $\left.^{-}\right)$. If the sense of agency is critical, we would expect participants to prefer the "safe" target in the latter conditions.

We also designed Experiment 2 to provide a second test of the SPE hypothesis. To that end, we compared conditions in which the trial outcome included clamped cursor feedback $\left(\mathrm{SPE}^{+}\right)$or did not include this feedback $\left(\mathrm{SPE}^{-}\right)$. This feedback, when provided, was always presented at the same location midway between the two targets, independent of their target choice. Based on previous work with clamped feedback (Morehead et al., 2017; Kim et al., 2018), we assumed that this signal would automatically be treated by the motor system as an SPE, driving sensorimotor adaptation. However, given the results of Experiment 1, we expected that the presence or absence of SPE would not influence choice behavior.

We first verified that clamped feedback, even if only presented at the end of the movement, was sufficient to produce adaptation (see Materials and Methods, Clamp-Only Experiment). Despite being informed about the nature of the clamped feedback and instructed to ignore it, robust adaptation was observed when the clamped feedback was presented: During the clamp block, the heading angle for each target shifted in the opposite direction of the cursor and an aftereffect was observed (Fig. 4B). A $t$ test of the baseline-subtracted final heading angle revealed the aftereffect being significantly $>0\left(t_{(13)}=4.65, p<0.001\right)$. Moreover, these effects were absent if the feedback was delayed by $2 \mathrm{~s}\left(t_{(13)}=\right.$ $-0.19, p=0.85)$, providing further evidence that this type of feedback is treated like an SPE by the motor system and causes 
A

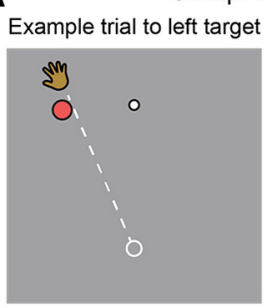

Clamped Feedback

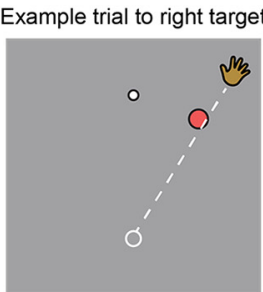

B

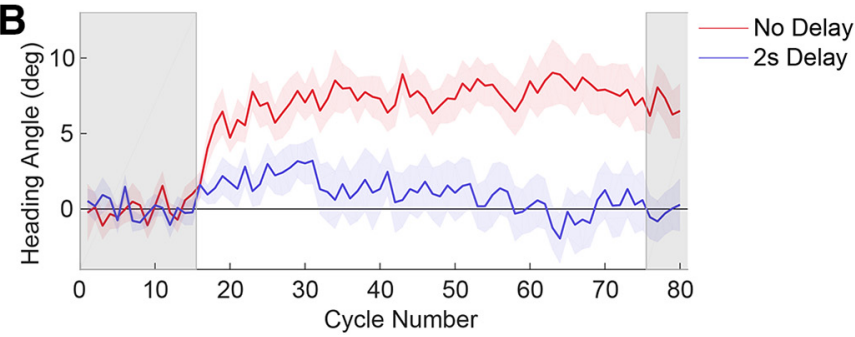

Figure 4. Clamp-only experiment showing sensorimotor adaptation from clamped feedback, but only if the feedback is immediate. $\boldsymbol{A}$, Participants were instructed to reach toward the single target. Clamped feedback would always appear straight ahead at the end of the reach, regardless of the participant's heading angle. $\boldsymbol{B}$, Immediate clamped feedback ("No Delay") elicits a significant aftereffect in the expected direction for both targets. No aftereffect is observed when the clamped feedback is delayed by $2 s$ (" 2 s Delay"). Lines represent mean heading angle over participants and shaded regions around the lines represent \pm 1 SEM over participants. Gray regions represent baseline and aftereffect trials with no feedback.

A

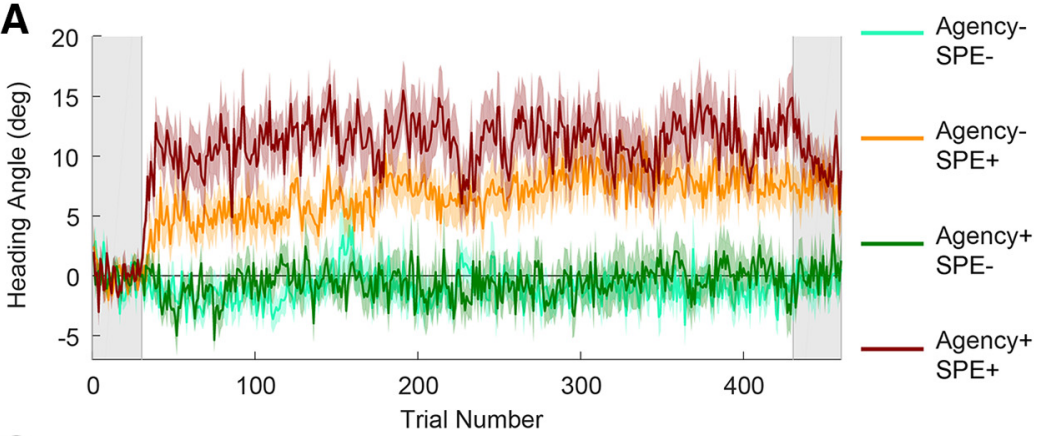

C

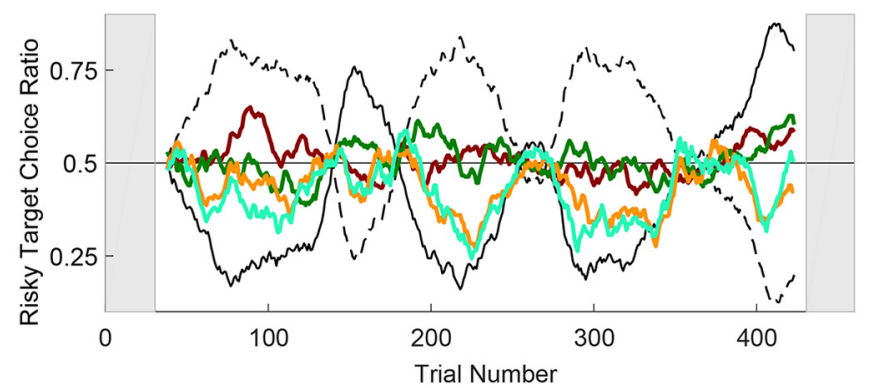

B

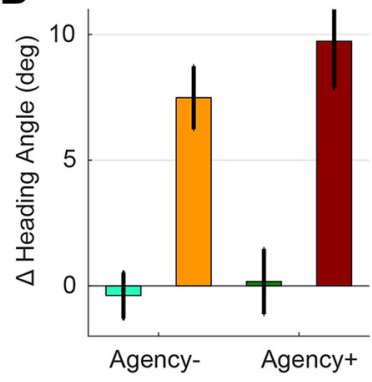

D

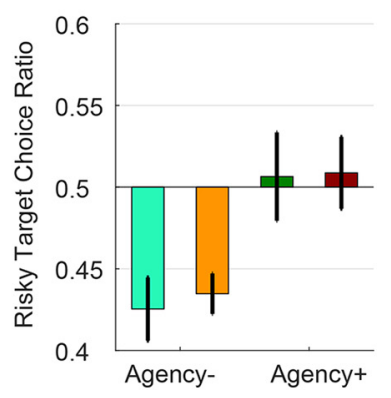

Figure 5. Sense of agency, but not presence of SPE, influences choice preference. $A$, Heading angle of reaches reveals the time course of adaptation. Heading angle for the $60^{\circ}$ target are flipped such that positive is in the direction of adaptation. Lines indicate mean heading angle over participants. Shaded regions around the lines represent \pm 1 SEM over participants. Gray regions represent

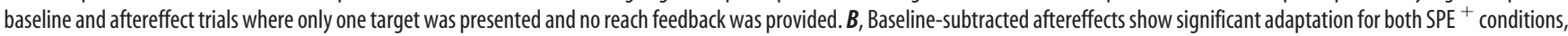
and none for the SPE ${ }^{-}$conditions. C, Group averaged choice behavior shows a bias toward the safe target for the Agency ${ }^{-}$conditions, and no bias for Agency ${ }^{+}$conditions. Colored lines indicate the proportion of choices made to the riskier target, averaged over participants in each condition (calculated over a 19-trial window moving average). The relative "riskiness" of target 1 and target 2 (determined by the predefined reward schedule) are shown for illustrative purposes (black solid and dashed lines). $\boldsymbol{D}$, Risk preference quantified as the ratio of trials where the riskier target was chosen over the total number of trials. Choice bias is influenced by a sense of agency, rather than SPE.

robust implicit learning (Held et al., 1966; Kitazawa et al., 1995; Honda et al., 2012; Brudner et al., 2016; Schween and Hegele, 2017).

Adaptation also occurred in response to the clamped feedback in the main experiment. During the choice trials, heading angle again shifted in the opposite direction of the cursor (Fig. 5A), and there was a pronounced aftereffect (Fig. 5B). (Such an accumulation of adaptation leading to an aftereffect would not occur in Experiment 1, as errors were presented on both sides for each target). These effects were not observed for the groups in which the cursor was never presented (SPE-groups). A two-way ANOVA comparing the heading angle in the aftereffect block to the baseline block revealed a main effect of SPE $\left(F_{(1,76)}=40.7, p<\right.$ $0.001)$, but no effect of agency $\left(F_{(1,76)}=1.05, p=0.31\right)$ or an interaction $\left(F_{(1,76)}=0.38, p=0.54\right)$. We note that the magnitude of the adaptation was numerically larger for the SPE group who were told they controlled the trial outcome. Although this may indicate that adaptation is influenced by a sense of agency, the participants in the Agency ${ }^{+}$group chose the risky target more often (see below), experienced more "miss trials," and thus received more SPEs.

Having established that the clamped feedback was an effective SPE, we next asked whether choice behavior was influenced by the presence of an SPE, a sense of agency, or an interaction of these variables. When participants were led to believe that the absence of reward was due to an action execution error, they did not show the same risk averse ("safe") bias compared with when they were told that the absence of reward reflected a probabilistic property of the target. As can be seen in Figure $5 C, D$, the Agency ${ }^{-}$groups tracked the "safe" target, whereas the Agency ${ }^{+}$ groups showed no consistent bias in their choice behavior. In contrast, the presence of an SPE had no influence on choice behavior. A two-way ANOVA showed a main effect of agency $\left(F_{(1,76)}=13.83, p<0.001\right)$, but not feedback type $\left(F_{(1,76)}=0.08\right.$, 

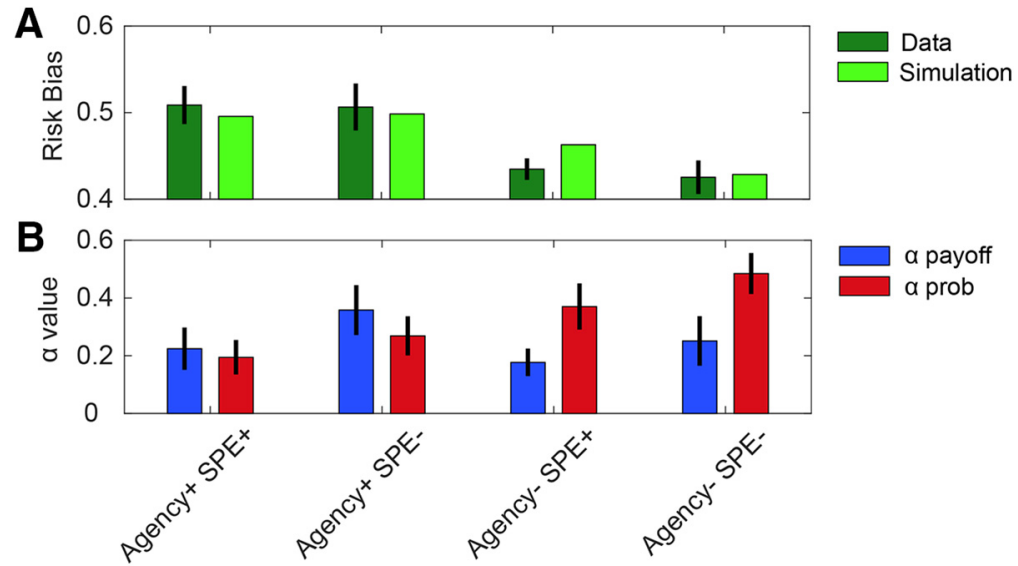

Figure 6. Agency model fits for Experiment 2.A, Simulations based on fitted parameters produce pattern of risk biases that are similar to those observed in the four conditions of Experiment 2. B, Fitted learning parameters $\left(\alpha_{\text {payoff }}\right.$ and $\left.\alpha_{\text {prob }}\right)$ for each condition. Agency ${ }^{+}$ conditions have a lower $\alpha_{\text {prob }}$ than Agency ${ }^{-}$conditions, consistent with the hypothesis that participants treat hit probabilities as less temporally dependent when they have a sense of agency. Error bars indicate \pm 1 SEM over participants.

$p=0.78)$, and there was no interaction between these variables $\left(F_{(1,76)}=0.03, p=0.87\right)$.

In summary, the results of Experiment 2 indicate that the presence of SPE, although leading to adaptation, was not sufficient to influence decision making. In contrast, variation in the sense of agency did influence choice behavior, with participants more likely to choose the risky target when they believed they were in control, at least to some degree, of the trial outcome.

\section{Model-based analysis of the agency hypothesis}

Experiment 2 was designed to examine whether choice behavior is affected when a sense of agency is explicitly manipulated, operationalized as the belief that outcomes are the result of motor performance. We hypothesized that a sense of agency would influence behavior by reducing the influence of temporal dependency of trial outcomes (Green et al., 2010). Specifically, if motor errors are assumed to reflect random noise in the Agency ${ }^{+}$conditions, recent hits and misses would not be informative about future hits and misses. In contrast, hit and miss outcomes are independent of the agent's motor accuracy in the Agency ${ }^{-}$conditions; thus, recent outcomes should provide useful information about future outcomes.

To evaluate whether this agency hypothesis could account for our observed behavior in Experiment 2, we developed a reinforcement learning model to capture how temporal dependency could influence choice behavior. In this model, the estimated hit probabilities $\hat{p}_{\mathrm{t}}(x)$ and payoffs $E_{\mathrm{t}}(x)$ for each target $x$ on trial $t$ are updated on a trial-by-trial basis, based on the differences between the actual and predicted outcomes (McDougle et al., 2016). The degree of temporal dependence is captured by two learning rate parameters, $\alpha_{\text {prob }}$ and $\alpha_{\text {payoff }}$, that correspond to the proportion that these estimates are updated based on the previous trial outcome as follows:

$$
\begin{gathered}
\delta_{\text {prob }, t}=r_{t}^{*}-\hat{p}_{t}(x) \\
\delta_{\text {payoff }, t}=r_{t}-E_{t}(x) \\
\hat{p}_{t+1}(x)=\hat{p}_{t}(x)+\alpha_{\text {prob }} \delta_{\text {prob }, t} \\
E_{t+1}(x)= \begin{cases}E_{t}(x)+\alpha_{\text {payoff }} \delta_{\text {payoff }, t}, & \text { if hit } \\
E_{t}(x), & \text { if } \text { miss }_{t}\end{cases}
\end{gathered}
$$$$
V_{t+1}(x)=\hat{p}_{t+1}(x) E_{t+1}(x)
$$

wendent on their rea believe the outcomes are deshould observe lower $\alpha$ compared with when they believe the outcomes are not dependent on reaching accuracy (Agency ${ }^{-}$ groups).

Estimated payoffs were updated in a similar manner to estimated probabilities. However, for payoffs, $r$ takes on values from 1 to 100 according to the observed payoff, and the update only occurs following hit trials. This conditional is a central component of the model, as it effectively separates trials in which outcomes are due to motor errors from trials that result in standard RPEs. $\alpha_{\text {payoff }}$ is fit as a free parameter for each participant and also reflects the degree of temporal dependence in payoffs. Because the payoff amounts were not dependent on hit accuracy, but rather a property of the target, we expected $\alpha_{\text {payoff }}$ to be approximately constant across all the experimental conditions.

Estimated target values $V(x)$ were transformed into probabilities using a standard softmax function. The inverse temperature parameter $(\tau)$ for the softmax was fit with one common value for all 80 participants in Experiment 2, resulting in 161 free parameters in total (one $\alpha_{\text {prob }}$ and $\alpha_{\text {payoff }}$ per participant, and one common $\tau$ ). Free parameter estimates were made using the fmincon function in MATLAB, which minimized the negative log likelihood of the choices for the parameters. The learning rates ( $\alpha_{\text {prob }}$ and $\alpha_{\text {payoff }}$ ) were bounded between 0 and 1 , and the inverse temperature parameter $(\tau)$ was bounded between 0.05 and 10 .

We fit the learning parameters, then generated choice data to simulate risk preferences. The agency model was capable of simulating the pattern of behavioral risk biases observed in Experiment 2 (Fig. 6A). Consistent with the predictions of the agency hypothesis, the groups that were told their reaching accuracy did not influence hit probability (Agency ${ }^{-}$groups) had a higher $\alpha_{\text {probability }}$ value than the groups that were told their reaching accuracy determined the hit outcomes (Agency ${ }^{+}$groups) (Fig. $6 B)$. A two-way ANOVA revealed a significant effect of agency on $\alpha_{\text {prob }}\left(F_{(1,76)}=7.85, p=0.01\right)$, no effect of $\operatorname{SPE}\left(F_{(1,76)}=1.82\right.$, $p=0.18)$, and no interaction between the two $\left(F_{(1,76)}=0.08, p=\right.$ 0.78 ). Also consistent with the agency hypothesis, a two-way ANOVA revealed no significant effects of agency on $\alpha_{\text {payoff }}\left(F_{(1,76)}=\right.$ $1.06, p=0.31)$, no effect of SPE $\left(F_{(1,76)}=1.93, p=0.17\right)$, and no interaction between the two $\left(F_{(1,76)}=0.16, p=0.69\right)$. 
These results support the hypothesis that differences in choice behavior across groups were mainly influenced by the degree to which they treated hit probabilities as being temporally dependent, with a belief of agency leading to more temporal independence.

\section{Discussion}

People are less sensitive to unrewarded outcomes when they are attributed to errors in action execution rather than action selection (McDougle et al., 2016). The main objective of this study was to evaluate different cues that could be used to solve this credit assignment problem. In earlier work, we had proposed a bottom-up hypothesis by which cerebellar-dependent SPEs were exploited by the reinforcement learning system, signaling the presence of an execution error (McDougle et al., 2016). By this model, SPEs provide a salient signal that the trial outcome should be attributed to the agent (i.e., execution error), rather than the chosen object (i.e., selection error). We tested this hypothesis in Experiment 1 by manipulating the strength of SPE and in Experiment 2 by presenting movement-irrelevant SPEs. In both cases, the results failed to support the hypothesis that SPE played a critical role in producing the observed bias in choice behavior. Instead, we found that the sense of agency had a significant effect on choice behavior, suggesting that the credit assignment problem may be solved in a more indirect, top-down manner.

\section{Salience of SPEs does not influence biases in choice behavior}

The strongest argument against the SPE hypothesis comes from Experiment 1. Here we compared conditions in which the feedback cursor was presented immediately at the end of the movement or after a 2 s delay. Previous work, as well as our clamp-only control experiment, has shown that a $2 \mathrm{~s}$ feedback delay strongly attenuates sensorimotor adaptation (Held et al., 1966; Kitazawa et al., 1995; Honda et al., 2012; Brudner et al., 2016; Schween and Hegele, 2017), presumably because the delay weakens the SPE. If SPE directly modulates choice preferences, then we expect participants to become more sensitive to unrewarded outcomes when the feedback was delayed. Although this effect was observed, a similar pattern was elicited when the intertrial interval was extended by $2 \mathrm{~s}$, even if the cursor feedback was immediate. Thus, the most parsimonious account of these results is that the time between successive choices, rather than SPE, decreased sensitivity to unrewarded outcomes.

Why might an increase in the intertrial interval change choice preferences? One hypothesis is that some form of iconic motor memory is strong when the interval is short (Adams and Dijkstra, 1966; Posner and Konick, 1966; Laabs, 1973; Annett, 1995; Miyamoto et al., 2014), leading the participants to believe they can correct the execution error. However, we found no evidence that participants showed a stronger adjustment in reach trajectories in the Immediate Feedback condition compared with when the feedback or intertrial interval was extended: The mean proportion of the error corrected on trials where feedback was artificially perturbed was $0.57(\mathrm{SE}=0.04)$ for the Immediate Feedback condition, 0.57 (0.08) for the Delayed Feedback condition, and 0.53 (0.04) for the Delayed Trials conditions. A one-way ANOVA on the regression between error and change in heading angle revealed no effect of group $\left(F_{(2,57)}=0.12, p=0.89\right)$. An alternative hypothesis is that the longer intertrial interval resulted in more time discounting of the potential rewards for each target (Frederick et al., 2002). This would have the effect of attenuating all choice biases, consistent with our findings.
The results of Experiment 2 provide further evidence against the SPE hypothesis. Here we used a method in which the SPE signal is not contingent on movement accuracy. Consistent with our previous work, this method was sufficient to produce adaptation in the reaching behavior of the participants. Nonetheless, choice biases were similar, regardless of whether this signal was present. Together, the results argue against a simple, bottom-up model in which an SPE signal is sufficient to attenuate value updates when the outcome error is attributed to a failure in motor execution.

\section{Choice biases are influenced by a sense of agency}

The results of the present study point toward a more top-down mechanism for solving the credit assignment required to differentiate execution and selection errors. This was most clearly observed in the results of Experiment 2, where sensitivity to unrewarded outcomes was reduced when the instructions emphasized that the participants had some degree of agency in determining the outcome, with agency operationalized as the belief that outcomes are dependent on one's motor performance. Similarly, Green et al. (2010) found that choice behavior could be dramatically altered by instructing participants that the trial outcome was either determined by the computer or contingent on movement execution. Computationally, they suggested that people assume weaker temporal dependence between successive events when the outcomes depend on motor output, given that errors from motor noise are assumed to be random. Properties of the object, however, may be more temporally dependent (e.g., the target with the high payoff on the previous trial is likely to yield a high payoff on the next trial).

In modeling the data from Experiment 2, we adopted an operational definition of agency introduced by Green et al. (2010), namely, that a sense of agency will cause choices to be more temporally independent. Consistent with the agency hypothesis, the fits showed that participants in conditions of high agency were less likely to behave as though hit outcomes were temporally dependent. In other words, by treating execution errors as though they were random events and unlikely to occur again, they were more likely to choose the target with the higher expected payoff. Participants in the low agency condition, however, were more likely to behave as though misses were a property of the target and, therefore, were biased to avoid the target which resulted in more misses.

We note that, in our earlier study (McDougle et al., 2016), we had included a similar manipulation of a sense of control, informing participants that the position of the feedback cursor was either dependent or independent of their movement. Contrary to the current results, we observed no effect of agency on choice behavior when an SPE-like signal was present. However, the feedback cursor still appeared near the selected target, either as veridical feedback or in a slightly shifted position. It is possible that, despite the instructions, the correlation between their movements and sensory feedback may have led the participants to believe, implicitly or explicitly, that they could control the reward outcomes. The clamped feedback used in Experiment 2 avoids this problem because the feedback was spatially independent of the movement.

A similar explanation may also account for the betweenexperiment differences in choice behavior observed in conditions in which the participants were instructed to believe they were in control of the trial outcomes. Although the reward schedules were identical, the participants in Experiment 1 exhibited a stronger bias for the risky target than the participants in Experiment 2. 
This was verified in a post hoc analysis, restricted to the Immediate Feedback condition in Experiment 1 and the two Agency ${ }^{+}$ groups in Experiment $2\left(t_{(58)}=4.25, p<0.001\right)$. The main difference between these conditions was that endpoint reach feedback was provided in Experiment 1, but not Experiment 2. The endpoint feedback not only provided a salient cue for motor performance, but also signaled a strong causal relationship association between trials in which the cursor hit the target and the participant being awarded points. These signals would likely increase the participants' confidence that the outcomes reflect their motor performance, increasing their sense of agency and, thus, produce a stronger risk bias.

In addition to an overall sense of agency, there is another way in which reach feedback might influence choice behavior. The presence of reach feedback results in salient, "near miss" trials. These have been shown, at least under some conditions, to produce similar hemodynamic responses as are observed with rewarded trials (Clark et al., 2009). Treating these near miss outcomes as rewarding, even if only slightly, would result in a stronger risk bias when reach feedback was present in Experiment 1, but not in Experiment 2.

\section{Mechanistic considerations for the modulation of reinforcement learning by execution errors}

As noted in the Introduction, distinguishing between action execution and action selection errors is important to optimize choice behavior. Knocking over a cup of coffee should not make us dislike coffee, even though we failed to obtain an expected reward. Current models of decision making tend to be based on tasks in which execution errors are absent; yet these systems evolved in organisms in which outcomes almost always reflected the interaction of processes involved in selection and execution. We can envision two ways in which an execution error might gate value updating. The negative RPE signals associated with unsuccessful outcomes might be attenuated. Or the operation by which these signals modify value representations might be disrupted.

The SPE hypothesis was motivated, in part, by consideration of recently described projections between the cerebellum and basal ganglia (Hoshi et al., 2005; Bostan et al., 2010; Chen et al., 2014) and association areas of the cerebral cortex implicated in value representation (O'Doherty, 2004; Choi et al., 2012). We hypothesized that execution error signals, which evolved to keep the sensorimotor system calibrated, may have come to be exploited by the reinforcement learning system. However, the results from the current experiments provide strong evidence against this simple, bottom-up account of how a decision-making system might distinguish between action execution and action selection errors.

Instead, the current results suggest that this gating process is driven by explicit knowledge about the source of errors, information that is dependent on a sense of agency. This contextual knowledge could have a direct influence on how RPEs are computed or used to update value representations. The recruitment of working memory (Collins et al., 2017) and explicit knowledge about task contingencies ( $\mathrm{Li}$ et al., 2011) have been shown to affect hemodynamic signatures of RPEs in ventral striatum and ventromedial prefrontal cortex. In a similar fashion, top-down knowledge about the success or failure of action execution could provide a similar modulatory signal, either to a system generating RPEs or using this information to update value representations. By using responses that offer the possibility of execution errors, it should be possible to use fMRI to identify neural loci that are sensitive to the intersection of action execution and action selection.

\section{References}

Adams JA, Dijkstra S (1966) Short-term memory for motor responses. J Exp Psychol 71:314-318. CrossRef Medline

Annett J (1995) Motor imagery: perception or action? Neuropsychologia 33:1395-1417. CrossRef Medline

Bostan AC, Dum RP, Strick PL (2010) The basal ganglia communicate with the cerebellum. Proc Natl Acad Sci U S A 107:8452-8456. CrossRef Medline

Brudner SN, Kethidi N, Graeupner D, Ivry RB, Taylor JA (2016) Delayed feedback during sensorimotor learning selectively disrupts adaptation but not strategy use. J Neurophysiol 115:1499-1511. CrossRef Medline

Caraco T, Martindale S, Whittam TS (1980) An empirical demonstration of risk-sensitive foraging preferences. Anim Behav 28:820-830. CrossRef

Chen CH, Fremont R, Arteaga-Bracho EE, Khodakhah K (2014) Short latency cerebellar modulation of the basal ganglia. Nat Neurosci 17:17671775. CrossRef Medline

Choi EY, Yeo BT, Buckner RL (2012) The organization of the human striatum estimated by intrinsic functional connectivity. J Neurophysiol 108: 2242-2263. CrossRef Medline

Clark L, Lawrence AJ, Astley-Jones F, Gray N (2009) Gambling near-misses enhance motivation to gamble and recruit win-related brain circuitry. Neuron 61:481-490. CrossRef Medline

Collins AG, Ciullo B, Frank MJ, Badre D (2017) Working memory load strengthens reward prediction errors. J Neurosci 37:4332-4342. CrossRef Medline

Dayan P, Niv Y (2008) Reinforcement learning: the good, the bad and the ugly. Curr Opin Neurobiol 18:185-196. CrossRef Medline

Dienes Z (2014) Using Bayes to get the most out of non-significant results. Front Psychol 5:781. CrossRef Medline

Frederick S, Loewenstein G, O'Donoghue T (2002) Time discounting and time preference: a critical review. J Econ Lit 40:351-401. CrossRef

Green CS, Benson C, Kersten D, Schrater P (2010) Alterations in choice behavior by manipulations of world model. Proc Natl Acad Sci U S A 107:16401-16406. CrossRef Medline

Hartmann MN, Hager OM, Tobler PN, Kaiser S (2013) Parabolic discounting of monetary rewards by physical effort. Behav Processes 100:192-196. CrossRef Medline

Held R, Efstathiou A, Greene M (1966) Adaptation to displaced and delayed visual feedback from the hand. J Exp Psychol 72:887-891.

Honda T, Hirashima M, Nozaki D (2012) Habituation to feedback delay restores degraded visuomotor adaptation by altering both sensory prediction error and the sensitivity of adaptation to the error. Front Psychol 3:540. CrossRef Medline

Hoshi E, Tremblay L, Féger J, Carras PL, Strick PL (2005) The cerebellum communicates with the basal ganglia. Nat Neurosci 8:1491-1493. CrossRef Medline

Kahneman D, Tversky A (1979) Prospect theory: an analysis of decision under risk. Econometrica 47:263-291. CrossRef

Kim HE, Morehead JR, Parvin DE, Moazzezi R, Ivry RB (2018) Invariant errors reveal limitations in motor correction rather than constraints on error sensitivity. Commun Biol 1:19. CrossRef

Kitazawa S, Kohno T, Uka T (1995) Effects of delayed visual information on the rate and amount of prism adaptation in the human. J Neurosci 15: 7644-7652. CrossRef Medline

Laabs GJ (1973) Retention characteristics of different reproduction cues in motor short-term memory. J Exp Psychol 100:168-177. CrossRef Medline

Landy MS, Trommershäuser J, Daw ND (2012) Dynamic estimation of task-relevant variance in movement under risk. J Neurosci 32:1270212711. CrossRef Medline

Li J, Delgado MR, Phelps EA (2011) How instructed knowledge modulates the neural systems of reward learning. Proc Natl Acad Sci U S A 108:5560. CrossRef Medline

McDougle SD, Boggess MJ, Crossley MJ, Parvin D, Ivry RB, Taylor JA (2016) Credit assignment in movement-dependent reinforcement learning. Proc Natl Acad Sci U S A 113:6797-6802. CrossRef Medline

Miyamoto YR, Wang SX, Brennan AE, Smith MA (2014) Distinct forms of implicit learning that respond differentially to performance errors and sensory prediction errors. Paper presented at the conference on Transla- 
tional and Computational Motor Control, Washington DC, District of Columbia.

Morehead JR, Taylor JA, Parvin DE, Ivry RB (2017) Characteristics of implicit sensorimotor adaptation revealed by task-irrelevant clamped feedback. J Cogn Neurosci 29:1061-1074. CrossRef Medline

Niv Y, Edlund JA, Dayan P, O’Doherty JP (2012) Neural prediction errors reveal a risk-sensitive reinforcement-learning process in the human brain. J Neurosci 32:551-562. CrossRef Medline

O'Doherty JP (2004) Reward representations and reward-related learning in the human brain: insights from neuroimaging. Curr Opin Neurobiol 14:769-776. CrossRef Medline

Oldfield RC (1971) The assessment and analysis of handedness: the Edinburgh Inventory. Neuropsychologia 9:97-113. CrossRef Medline

Pelli DG (1997) The VideoToolbox software for visual psychophysics: transforming numbers into movies. Spat Vis 10:437-442. CrossRef Medline

Posner MI, Konick AF (1966) Short-term retention of visual and kinesthetic information. Organ Behav Hum Perform 1:71-86. CrossRef

Rescorla RA, Wagner AR (1972) A theory of Pavlovian conditioning: variations in the effectiveness of reinforcement and nonreinforcement. In: Classical conditioning, Vol II: Current research and theory (Black AH, Prokasy WF, eds), pp 64-99. New York, NY: Appleton-Century-Crofts.

Rouder JN, Speckman PL, Sun D, Morey RD, Iverson G (2009) Bayesian $t$ tests for accepting and rejecting the null hypothesis. Psychon Bull Rev 16:225-237. CrossRef Medline
Schultz W (2016) Dopamine reward prediction error coding. Dialogues Clin Neurosci 18:23-32. Medline

Schultz W, Dayan P, Montague PR (1997) A neural substrate of prediction and reward. Science 275:1593-1599. CrossRef Medline

Schween R, Hegele M (2017) Feedback delay attenuates implicit but facilitates explicit adjustments to a visuomotor rotation. Neurobiol Learn Mem 140:124-133. CrossRef Medline

Trommershäuser J, Maloney LT, Landy MS (2008) Decision making, movement planning, and statistical decision theory. Trends Cogn Sci 12:291297. CrossRef Medline

Tseng YW, Diedrichsen J, Krakauer JW, Shadmehr R, Bastian AJ (2007) Sensory prediction errors drive cerebellum-dependent adaptation of reaching. J Neurophysiol 98:54-62. CrossRef Medline

Walton ME, Kennerley SW, Bannerman DM, Phillips PE, Rushworth MF (2006) Weighing up the benefits of work: behavioral and neural analyses of effort-related decision making. Neural Netw 19:1302-1314. CrossRef Medline

Wolpert DM, Ghahramani Z, Jordan MI (1995) An internal model for sensorimotor integration. Science 269:1880-1882. CrossRef Medline

Wu SW, Delgado MR, Maloney LT (2009) Economic decision-making compared with an equivalent motor task. Proc Natl Acad Sci U S A 106 6088-6093. CrossRef Medline

Wu SW, Delgado MR, Maloney LT (2011) The neural correlates of subjective utility of monetary outcome and probability weight in economic and in motor decision under risk. J Neurosci 31:8822-8831. CrossRef Medline 